\title{
Acknowledgment to reviewers
}

๑ The Physiological Society of Japan and Springer Japan KK, part of Springer Nature 2018

We gratefully acknowledge the contributions of:

Abe, Chikara
Abe, Takashi
Aibiki, Mayuki
Akaike, Toru
Akimoto, Takayuki
Akita, Tenpei
Alijanpour, Sakineh
Amano, Akira
Ando, Soichi
Anzai, Naohiko
Arafune, Tatsuhiko
Asano, Shinji
Ashihara, Takashi
Aso-Someya, Nami
Behringer, Michael
Bjorklund, Geir
Brassard, Patrice
Chen, Dongshi
Daimon, Masao
Davis, Scott
Descarreaux, Martin
Dezaki, Katsuya
Duchamp, Claude
Ebraheim, Nabil
Egawa, Tatsuro
Fujii, Naoto
Fujii, Nobuharu
Fujiki, Nobuhiro
Fujimoto, Naoki
Fujino, Hidemi
Fujita, Takayuki
Fukada, Toshiyuki
Fuke, Soichiro
Fukuoka, Yoshiyuki
Funahashi, Toshiya
Goto, Tsuyoshi
Hamaoka, Takafumi
Hashimoto, Takeshi

Abe, Chikara

Aibiki, Mayuki

Akaike, Toru

Akita, Tenpei

Alijanpour, Sakineh

Amano, Akira

Anzai, Naohiko

Arafune, Tatsuhiko

Asano, Shinj

Ashihara, Takashi

Aso-Someya, Nami

Behringer, Micha

Brassard, Patrice

Chen, Dongshi

Daimon, Masao

Davis, Scott

Descarreaux, Martin

Dezaki, Katsuya

Duchamp, Claude

Ebraheim, Nabi

Egawa, Tatsuro

Fujii, Naoto

Fujii, Nobuharu

Fujiki, Nobuhiro

Fujimoto, Naok

Fujino, Hidem

Fujita, Takayuki

Fukada, Toshiyuki

Fuke, Soichiro

Fukuoka, Yoshiyuki

Funahashi, Toshiya

Hamaoka, Takafumi

Hashimoto, Takeshi

\author{
Hashitani, Hikaru \\ Hatano, Ryo \\ Hattori, Yuichi \\ Herzog, Erik D. \\ Hieda, Michinari \\ Higaki, Yasuki \\ Higashida, Haruhiro \\ Higuchi, Keiichi \\ Higuchi, Sayaka \\ Hirano, Katsuya \\ Hisatome, Ichiro \\ Hori, Etsuo \\ Horiuchi, Masahiro \\ Huang, Yizhong \\ Iemitsu, Motoyuki \\ Iimura, Kaori \\ Ikari, Akira \\ Ikeda, Masayuki \\ Ikemura, Tsukasa \\ Inagaki, Masashi \\ Ishibashi, Kenichi \\ Ishihara, Akihiko \\ Ishii, Hisayoshi \\ Ishii, Kuniaki \\ Itoi, Hirosumi \\ Iwasa, Masayo \\ Iwase, Satoshi \\ Izawa, Tetsuya \\ Kakinuma, Yoshihiko \\ Kamijo, Yoshi-Ichiro \\ Kamiya, Atsunori \\ Kano, Yutaka \\ Kashiwadani, Hideki
}

Kato, Nobuo

Kaur, Keerat

Kawai, Yasuaki

Kawano, Fuminori

Kim, Hee Young

Kirimoto, Hikari

Kisaka, Tomohiko

Kitamura, Tadahiro

Koba, Satoshi

Kollmar, Richard

Kondo, Kenji

Kondo, Tetsuri

Konishi, Masato

Koshiya, Naohiro

Koyama, Katsuhiro

Koyama, Natsu

Kuo, Yu-Min

Kurihara, Takashi

Kuwana, Shun-ichi

Kwon, Sunoh

Le, Anh

Li, Meihua

Li, Yong

Liang, Nu-Chu

Liu, Haizhou

Liu, Yu-Fan

Lo, Amy

Low, David

Maruyama, Takashi

Masamoto, Kazuto

Masubuchi, Satoru

Masuda, Kazumi

Masuda, Shinya

Masuki, Shizue

Masutani, Satoshi

Matsui, Yoshiro

Matsukawa, Kanji

Matsunaga, Masahiro 
Matsuura, Hiroshi

Medeiros, Liciane

Mikami, Yoshinori

Minokoshi, Yasuhiko

Mitsushima, Dai

Miura, Shinji

Miura, Tetsuji

Miyachi, Motohiko

Miyai, Kazumasa

Miyata, Hirofumi

Miyazaki, Mitsunori

Miyoshi, Goichi

Mizumura, Kazue

Mohri, Satoshi

Mori, Masayuki (Kyoto)

Mori, Masayuki (Matsumoto)

Mukkamala, Ramakrishna

Muramatsu, Ken

Nagamori, Shushi

Nagatomi, Ryoichi

Naito, Hisashi

Nakajima, Kenichiro

Nakanishi, Atsuko

Nakashima, Noriyuki

Nakata, Hiroki

Nakayama, Shinsuke

Neyroud, Daria

Nishida, Motohiro

Nishijima, Takeshi

Nowak, Robert

Numata, Tomohiro

Obata, Koji

Ogura, Yuji

Ohnishi, Norikazu

Oiki, Shigetoshi

Okada, Yasumasa

Okada, Yasunobu

Okamoto, Shiki

Okamoto, Takanobu

Okazaki, Kazunobu
Okutsu, Mitsuharu

Olcese, James

Onaka, Tatsushi

Onimaru, Hiroshi

Ono, Kyoichi

Ono, Yusuke

Ootsuka, Youichirou

Ortega de Mues, Arantxa

Otsuki, Takeshi

Ozawa, Hitoshi

Pearson, James

Porta, Alberto

Quindry, John

Rashidy-Pour, Ali

Rustamov, Nabi

Sadato, Norihiro

Sakai, Tetsuro

Sakamoto, Kenji

Sakata, Susumu

Saku, Keita

Sakurai, Kenichi

Sata, Yusuke

Sei, Hiroyoshi

Seo, Yoshiteru

Shea, Graham

Shibamoto, Toshishige

Shibata, Shigeki

Shiina, Takahiko

Shimayoshi, Takao

Shimizu, Shuji

Shioya, Takao

Somlyo, Avril V.

Sudo, Mizuki

Suganami, Takayoshi

Sugawara, Jun

Sugimachi, Masaru

Sugimoto, Naotoshi

Sugiura, Seiryo

Sugiura, Takao

Sun, Yao
Sunagawa, Genshiro

Suzuki, Atsuko

Suzuki, Katsuhiko

Suzuki, Yoshiro

Takahashi, Eiji

Takamata, Akira

Takanari, Hiroki

Takayanagi, Yuki

Takeuchi, Ayako

Tamaki, Hiroyuki

Tanaka, Hikaru

Tanaka, Kunihiko

Tanaka, Reiko

Tanaka, Satoshi

Taniguchi, Shun'ichiro

Tanimura, Yuko

Tarumi, Takashi

Tokizawa, Ken

Torres-Aleman, Ignacio

Tricoli, Valmor

Uchida, Kunitoshi

Uchida, Sae

Uchida, Yuki

Ueno, Hiromichi

Wüst, Rob

Waki, Hidefumi

Wang, Guan

Watanabe, Nobuhiro

Yada, Toshihiko

Yaku, Hitoshi

Yamamoto, Yoshimichi

Yamamura, Aya

Yamashita, Akira

Yasoshima, Yasunobu

Yoshimura, Mitsuhiro

Yoshina, Sawako

Yoshitake, Yasuhide

Yoshizato, Hideo

Zhang, Haiying

Zwetsloot, Kevin 